\title{
EL DESARROLLO DE ENFERMERÍA EN AMÉRICA LATINA: UNA MIRADA ESTRATÉGICA
}

Maricel Manfredi*

\begin{abstract}
La enfermería en América Latina enfrenta un número de graves desafíos que afectan significativamente la contribución que su personal puede hacer en pro de la salud de la población que sirve.

Este artículo presenta una vista general de enfermería como un componente importante del sistema de salud de América Latina a través de la perspectiva y la orientación que provee el planeamiento estratégico.

La autora haciendo un análisis de la situación de enfermería en la región identifica varios problemas creando luego una visión para el futuro. El artículo contempla las posibilidades de desarrollar e implementar proyectos y planes de acción dirigidos a resolver los problemas más urgentes. Considera al pensamiento estratégico como un medio posible de reflexión para enfermería y de acrecentar al máximo su presencia y contribución a la solución de los problemas de salud de la presente década y del próximo siglo.
\end{abstract}

\section{Introducción}

La elaboración de este artículo para la primera edición de la Revista Latinoamericana de Enfermería coincide con la celebración de los 90 años de fundación de la Organización Panamericana de la Salud de las Américas. Noventa años de esfuerzo conjunto, Organizacióngobierno miembros, para la construcción de mejores condiciones de vida y de salud para los pueblos de las Américas.

Enfermería ha estado presente colaborando en la construcción de la historia y misión de la Organización desde el comienzo de esta a principios de este siglo, siempre manteniendo el convencimiento que su acción está orientada al servicio de aquellas poblaciones que más lo necesitan, constituyéndose en pilar fundamental para la implementación de la estrategia de atención primaria y conformando parte fundamental del recurso humano que conforman los equipos de trabajo dentro de los sistemas locales de salud.

La cooperación técnica en el área de enfermería ha sido muy bien estudiada y profundizada en una tesis de grado de doctorado de Dra. Alina Souza ${ }^{1}$ y en el documento de "Cuarenta años de cooperación técnica de enfermería en la OPS"2, por lo cual en este artículo me permitiré presentar a Uds. Una visión de enfermería de la región tomando algunos elementos de la planificación estratégica para hacer dicho análisis. Trataré entonces de analizar muy someramente la situación de enfermería de la región, algunos problemas que se han identificado, dar una visión global del futuro y sugerir la construcción de algunos proyectos y planes de acción.

\footnotetext{
*Asesora Regional para Educación da Enfermería. Programa Regional de Recursos Humanos. Organización Panamericana de la Salud, Wash. D.C. Agosto 1992. Artículo adaptado de la presentación hecha al Congreso de Sociedades Científicas de Enfermería en Chile en Agosto de 1992.
} 


\section{La salud y el desarrollo en América Latina}

Nos acercamos al Siglo XXI, en un mundo lleno de incertidumbre, imprevistos $y$ desconocimiento del futuro; arrastrando, al mismo tiempo, muchos de los problemas del pasado que todos Uds. conocen y que tienen que ver con el contexto político, social y económico en que están inmersos nuestros pueblos de América Latina.

Las consecuencias sociales de la crisis que vive la región son preocupantes ya que esencialmente han significado un acrecentamiento de la desigualdad y un crecimiento de la pobreza, lo cual se ha visto magnificado tanto por las disrupciones originadas por la urbanización masiva como por el crecimiento demográfico ${ }^{3}$. El impacto de los ajustes económicos ha deteriorado el desarrollo humano incluso en algunos países donde este último era un factor importante dentro de sus procesos políticos y sociales.

La crisis y el ajuste ( $y$, en ocasiones, peor el ajuste que la crisis) han agudizado algunos de salud en la región y están contribuyendo a generar problemas nuevos de enorme gravedad ${ }^{4}$. En síntesis, ha habido una enorme reducción y/o estancamiento en el monto de los recursos disponibles para el desarrollo y la operación de los servicios de salud. Esto se ha manifestado en las limitaciones de inversiones destinadas al saneamiento básico y el reemplazo, mantenimiento, y conservación de equipo y planta física. Asimismo los patrones de enfermedad en nuestros países están experimentando una transición epidemiológica hacia enfermedades de países desarrollados sin haber resuelto los problemas relacionados con el subdesarrollo y la pobreza. Ejemplos claros de esto son la Malaria, el Cólera y el SIDA. La inesperada epidemia de cólera en la mayoría de los países latinoamericanos son un ejemplo del deterioro de las condiciones de salud de nuestros países especialmente de la salud ambiental y su relación con la pobreza.

En especial se destaca el efecto de la crisis en la fuerza de trabajo en el sector salud, en lo que se refiere a la política de empleo y de ajustes salariales con la consiguiente fuga del personal. En enfermería esto se ha traducido en desempleo, subempleo, pobres condiciones de trabajo y, por onde, la mala utilización del recurso. La configuración heterogénea de la fuerza de trabajo en enfermería y la poca delimitación de roles y espacios de práctica la hace más vulnerable en estos momentos de crisis pues, según apreciaciones de otros, se piensa que se puede contar con un recurso menos costoso. La crisis del sector público ha llevado al desarrollo, muchas veces desmesurado del sector privado originando el en algunos países el drenaje de la fuerza de trabaja más calificada a este sector y en otros países el de personal menos calificado. En cuanto al sector formativo la crisis de los 80 ha agudizado las posibilidades de mantener un cuerpo docente bien calificado y remunerado, y unas instalaciones físicas adecuadas a las necesidades educacionales actuales. En profesiones como enfermería los efectos de la crisis están produciendo en algunos países disminución en las matrículas e las Escuelas de Enfermería, problema que tendrá grandes repercusiones en la atención de salud si no se toman las medidas correctivas necesarias. De agudizarse esta situación la posibilidad de contar con enfermeras bien calificadas en nuestros países se convertiría en un lujo.

Con este panorama general, la década de los 90 representa un reto para rectificar los daños producidos en el desarrollo humano, constituyendo un momentum para lograr las metas esenciales para el año 2000. Las respuestas a este reto requieren de la movilización de mayores recursos a nivel nacional e internacional $y$, en muchas instancias, reordenamiento de la distribución de los presupuestos así como cambios en los enfoques de asistencia de salud y en las orientaciones educacionales que permitan soluciones creativas al momento de crisis. 


\section{Situación actual de las instituciones formadoras de recursos humanos en enfermería y su relación con la salud de la población}

La carrera de enfermería en nuestros países dentro de la universidad es relativamente reciente (1940) y su ubicación ha estado determinada por estructuras hegemónicas que en muchas ocasiones no le han permitido actuar en una forma más independiente. Aún existen en el continente la formación de enfermeras a nivel hospitalario y/o técnico ligadas a los ministerios de salud o instituciones hospitalarias. Su traslado a instituciones de nivel superior se encuentran determinadas a decisiones influenciadas por los sectores dominantes de salud. En una investigación sobre Educación de Enfermería realizada en el año 84, se señalaba que sólo el 69\% de las escuelas que participaron en el estudio eran universitarias o estaban ascritas a la universidad ${ }^{5}$.

Los procesos de formación de la enfermera son muy heterogéneos; se pueden separar en dos grupos. Uno, el de las instituciones formadoras que han tenido cambios trascendentales y cuyas orientaciones curriculares en la actualidad representan transformaciones medulares en cuanto a nuevos enfoques volcados hacia la salud de la población. Otro, el de las instituciones que han permanecido al margen de los cambios y que mantienen estructuras tradicionales con enfoques educativos centrados en la atención individual y a nivel hospitalario con mayor énfasis en el desarrollo de la habilidad de atención a pacientes y enfoque más superficial de los problemas prevalentes de salud en el país, de los problemas sociales y de acciones dirigidos a promoción y prevención.

La formación del auxiliar de enfermería sigue estando ligada mayoritariamente a los ministerios de salud o instituciones dedicadas a la preparación de nivel técnico y/o vocacional. Sin embargo se vislumbra en algunos países el crecimiento de instituciones privadas con fines de lucro con poca o ninguna ingerencia de los organismos acreditados para evaluación de dicha formación.

Por otro lado a existido poca aceptación por parte del profesional de la enfermería de analizar más a profundidad la posibilidad de ofrecer a la auxiliar nuevas perspectivas de proseguir hacia la formación profesional, utilizando metodologías educacionales innovativas y basándose en teorías educacionales que permitan dar crédito a experiencias pasadas debidamente comprobadas.

La mayoría de las enfermeras en América Latina no tienen acceso a los procesos de educación continua, a oportunidades de actualización y de progreso en su educación que faciliten el cambio de actitudes y la adopción de nuevos perfiles educacionales. Además, estos procesos de educación continua no han estado vinculados directamente con la práctica en los servicios produciendo poco impacto para la transformación de los mismos.

\section{Práctica de enfermería}

Como se sabe, hasta ahora, la inserción de enfermería en los servicios de salud en América Latina ha estado vinculada al empleo en el sector público aunque en algunos países las crisis de este sector dentro de las estructuras políticas han abierto posibilidades para el trabajo de enfermería en organismos no gubernamentales o en el sector privado.

Aunque existe un movimiento para demostrar lo contrario, los espacios de práctica de enfermería siguen siendo limitados por razones de diversa índole que va desde los aspectos ya tratados de la crisis así como por la fuerte influencia de grupos hegemónicos dominantes. También por la poca visibilidad con que cuenta enfermería a nivel de decisiones así como por la falta de posicionamiento de las enfermeras frente a su quehacer y a la salud de la población. 
Al revisar varios documentos se encuentran evidentes contradicciones entre las declaraciones que reconocen el gran potencial de las enfermeras de hacer de realidad la OPS en los sistemas de salud y los espacios de participación que en realidad se le abren en muchos países.

Puede decirse que aun sigue siendo el nivel institucional el mayor empleador de enfermeras quizás por la misma estructura de los servicios pero, además, por la falta de entendimiento de su trascendental papel que tiene a nivel comunitario y que la historia de los servicios de salud de nuestros países lo demuestra.

Puedo parecer muy crítica pero creo que la administración y la organización de la atención de enfermería debe revitalizarse, debe democratizarse, debe descentralizarse, debe ayudar a las nuevas generaciones a abrirse una brecha para un mejor futuro. Se ha demostrado, por ejemplo, que las condiciones de trabajo, los incentivos, juegan un papel quizás más importante que el salario para la permanencia de las enfermeras en determinadas instituciones.

A pesar de todo esto, la enfermería ha demostrado desde un punto de vista mayoritario, un gran acercamiento hacia la población más necesitada y, a través de algunos modelos de atención innovadora, su gran capacidad de demostrar la diferencia. Como dijo el Dr. Mahler, exDirector de la OMS, el papel de la enfermera en conseguir cambios esenciales en los sistemas de salud basados en atención primaria es claro y evidente ${ }^{6}$.

La puesta en marcha de la nueva propuesta organizativa de los servicios de salud denominada sistemas locales de salud representa una forma de operacionalizar el concepto de atención primaria y es una respuesta interna del sector para lograr una mayor equidad, eficacia, y eficiencia de servicios dentro de las restricciones económicas de nuestros servicios. En muchos países enfermería está involucrada en este proceso ya que la posibilidad de demostrar la efectividad de este mismo depende en gran parte del recuso humano y dentro de éste de la labor de la enfermera para llegar a ofrecer salud a las poblaciones especialmente a aquellas que más la requieren.

\section{Investigación}

En un artículo publicado en el Journal of Advance Nursing, la Dra. Amelia Maglacas, actualmente Consultora en el área de enfermería a nivel internacional, dice: "las enfermeras en el sur de las Américas han aceptado e internalizado más rapidamente el reto que les impone Salud para Todos y la estrategia de atención primaria. Sin embargo por las numerosas restricciones a las que están abocadas dichas enfermeras en la educación y la práctica dichas innovaciones que se desarrollan con gran creatividad en el área de atención primaria no están sistemáticamente estudiadas o documentadas" ${ }^{7}$.

Creo que esta afirmación es cierta en el sentido que la investigación de enfermería en nuestro países sigue siendo escasa, poco conocida por la comunidad científica y el pueblo en general, pobremente utilizada y validada.

En el estudio de las tendencias de investigación en enfermería realizado con motivo del Primer Coloquio Panamericano de investigación de Enfermería, se encontró que las investigaciones tenían un énfasis clínico que posiblemente refleja no solo los modelos de preparación de profesionales que ha privilegiado los aspectos de la clínica, sino aspectos que se refuerzan cuando se analiza la distribución de investigaciones según sujetos de atención donde se evidencia que la mayoría de los estudios utilizó al individuo como objeto de estudio dejando a la familia y la comunidad en un segundo plano.

Por otra parte, el estudio reflejó que las investigaciones en su gran mayoría fueran de carácter descriptivo explicitando el desarrollo en que se encuentra la investigación de enfermería. Se comprobó, asimismo, la influencia de corrientes positivistas y de aquellas que enmarcan las conceptualizaciones dominantes en el de la salud. La utilización de la metodología descriptiva constituye un área crítica explicitando el desarrollo inicial en que encuentra la búsqueda y comprensión del 
objeto de enfermería.

La aplicación del conocimiento científico para el desarrollo de enfermería es un elemento crucial por lo que la investigación en enfermaría es necesaria para producir un impacto en el conocimiento y en la transformación de la atención de salud.

Existe en la actualidad en Latinoamérica un movimiento bastante grande por parte de enfermería para impulsar ;a investigación desde diferentes frentes especialmente a través de la Universidad y dentro del binomio docencia servicios, y apoyo de los mismos pero es quizás muy importante que las enfermeras aprendan a escribir, negociar y competir con proyectos de investigación que ofrecen "grants" a nivel nacional e internacional, así como a buscar espacios de publicación en revistas científicas latinoamericanas no necesariamente de enfermería.

La posibilidad de los Coloquios a nivel internacional abre una avenida para la presentación de resultados de investigaciones de intercambio y la posibilidad de cooperación mutua en las investigaciones de problemas comunes a uno o varios países. Sin embargo, debe seguirse trabajando en los países en la promoción de grupos de apoyo a la investigación que se conviertan en verdaderos promotores de la misma.

\section{El papel de las organizaciones no gubernamentales y centros colaboradores en el desarrollo de enfermería}

Las asociaciones de enfermeras, sociedades científicas, centros colaboradores de la OMS, se han organizado en esta última década, observándose una participación mucho más activa a nivel nacional e internacional. Estas han jugado un papel importante en actualizar, motivar y movilizar a las enfermeras hacia los cambios exigidos por las estrategias de atención primaria y sistemas locales de salud.

Muchas de las asociaciones se han fortalecido a través de proyectos promovidos por el Consejo Internacional de Enfermeras CIE, como son el de los servicios de atención primaria, liderazgo y legislación así como también a través de la ayuda sostenida dada por la Asociación Canadiense de Enfermeras para el desarrollo de proyectos específicos de enfermería en los países que han impulsado procesos de liderazgo en diversas áreas que tiene que ver con la labor gremial y técnica. En muchos países han mantenido un posicionamiento fuerte ante los problemas que afectan la salud de la población y del gremio mismo. Estos posicionamientos si bien a veces han creado conflictos con los grupos de poder han despertado un mayor conocimiento de la profesión. Es importante, sin embargo, que se mantengan mecanismos de cabildeo sistemático que permitía defender posiciones o presentar contribuciones para el mejoramiento de la salud de la población en forma constante.

A nivel internacional se han ganado espacios en foros a distintos niveles. La admisión en esta época de la Federación Panamericana de Enfermeras FEPPEN como observadora en los cuerpos directivos de la OPS. El nacimiento de la Asociación Latinoamericana de Facultades y Escuelas de Enfermería. Ambas asociaciones han impulsado proyectos a nivel internacional de acuerdo a sus propósitos. Entre ellos se pueden citar la reunión sobre el desarrollo de enfermería en los sistemas locales de salud organizados por FEPPEN y OPS en 1990, la I Conferencia Panamericana de Educación de Enfermería, desarrollada por ALADEFE, y así como los subsecuentes proyectos de FEPPEN y ALADEFE que concentran esfuerzos en el área de legislación, liderazgo, desarrollo de servicios, investigación y formación de recursos humanos.

Son pocas las sociedades científicas que existen en América Latina como parte de la Asociación de Enfermeras o como entidades separadas y creo que la labor de ellas en la actualización y en el impulso del desarrollo tecnológico en áreas determinadas es fundamental para mantener la calidad del cuidado y de la educación.

Existen además en esta región 7 centros colaboradores y dos más en proceso de ser nombrados. Cinco se encuentran en los Estados Unidos y dos en América Latina. 
Estos están en la Facultad de Enfermería de la Universidad de Illinois en Chicago, en la Facultad de Enfermería de la Universidad de Pennsylvania, en la Facultad de Enfermería de la Universidad de George Mason en Virginia, en la Facultad de Enfermería de la Universidad de Galveston en Texas, en la Facultad de Enfermería de la Universidad de California en San Francisco, en la Asociación de Facultades y Escuelas de Enfermería de Colombia en Bogotá y en la Facultad de Enfermería de la Universidad de São Paulo en Ribeirão Preto. Dos de los Centros en vía de reconocimiento como centros colaboradores están en Canadá en la Escuela de enfermería de la Universidad de MacMaster y en el Hospital del Monte Sinai en Toronto. Los Centros Colaboradores son considerados centros de excelencia en diversas áreas y su papel es el de contribuir a promulgar los mandatos de los cuerpos directivos a través de sus actividades internacionales y conjuntos con OMS/OPS.

Los centros colaboradores y las sociedades científicas son centros de excelencia para el desarrollo del conocimiento de modelos de práctica, contribuyen a expandir las oportunidades de intercambio, a fortalecer el trabajo colaborativo, a conformar núcleos de estudio y de investigación. Asimismo son un medio para expandir la cooperación técnica con los países al mismo tiempo que permiten la consecución de las metas de Salud para Todos a través de su contribución al desarrollo en los países de los programas prioritarios aceptados por los gobiernos de las Américas en reuniones internacionales.

Sin embargo es importante que se conozca a nivel de cada país lo que puede aportar cada una de estas sociedades y centros colaboradores al conocimiento de enfermería para así poder ser mejor utilizados. La creación de mecanismos de información y de difusión e intercambio de sus actividades es importante.

\section{Problemas críticos}

Dentro de una aproximación que necesita ser un poco más trabajada podemos identificar algunos problemas en el área de enfermería que ameritan ser discutidos no solo por las enfermeras sino dentro de una forma más amplia.

\section{Desbalance entre los diferentes niveles de personal salud}

- Disminución de la fuerza de trabajo profesional de enfermería en algunos países de las Américas debido a bajos salarios, condiciones de trabajo, status y ampliación de oportunidades en el mercado de trabajo para la mujer.

- Aumento del número de personal sin preparación dando atención de enfermería en detrimento de la calidad de servicios.

- Distribución geográfica in equitativa de los profesionales de enfermería y utilización inadecuada del mismo.

- Reducción de personal de enfermería por reducción de gastos en salud.

- Pobres condiciones de trabajo y falta de incentivos (no necesariamente económicos) que permitan mantener una motivación hacia su quehacer y una identificación positiva con la profesión. 


\section{Limitado apoyo a nivel nacional del componente enfermería}

- Limitada participación de enfermeras calificadas en niveles de toma de decisiones a nivel nacional.

- Poca participación de enfermería en el desarrollo de políticas de salud.

- Falta de estructuras de enfermería a nivel nacional y regional de acuerdo a los principios de descentralización.

- Poca visibilidad de enfermería hacia agendas de salud.

\section{Falta de visión global de los procesos educación de enfermería. Formación tradicional que no prepara para el cambio}

- Orientación curricular todavía muy centrada en el individuo en vez de la población en general y la enfermedad en lugar de la salud.

- Pobre integración docente asistencial. Las nuevas orientaciones curriculares no han contribuido a fortalecer cambios en la entrega de los servicios de la salud y atención de enfermería.

- Poca flexibilidad hacia nuevas posibilidades de desarrollo de una carrera de enfermería que incluya y rescate a los diferentes niveles de enfermería.

\section{Estancamiento en el liderazgo de enfermería}

- Orientaciones tradicionales hacia la administración de la atención de enfermería a nivel institucional y comunitario.

- Falta de orientación de los programas posbásicos de una conceptualización de enfermería dentro de una nueva visión de salud que incluya áreas como Economía, Ciencias PolíticoSociales, Administración.

- Limitadas posibilidades de especialización en áreas críticas a nivel de problemas básicos de salud y orientación de la atención.

- Programas de educación continua con visión restringida del proceso de educación permanente y que no favorecen la transferencia y mejoramiento de los servicios de salud.

\section{Limitada producción científica de enfermería que contribuya acrecentar el conocimiento en la área y a mejorar la atención de la salud.}

- Falta de mecanismos para incentivar la investigación a nivel nacional. 
- Falta de habilidad para la presentación de proyectos de investigación que pueden competir para financiamiento a nivel nacional e internacional.

- Poca utilización de los resultados de investigación en enfermería y pobres posibilidades de publicación.

- Limitada formación en el área de investigación en enfermería.

\section{Falta de legislaciones adecuadas a los cambios en la profesión y en la salud de los países}

- Legislaciones caducas que no permiten legalizar nuevas prácticas de enfermería.

- Falta de una acción concertada por parte de las asociaciones gremiales en la creación de una nueva imagen de enfermería y de un posicionamiento acerca de la contribución a la salud de los países, es decir la creación de una "visión de enfermería".

- Falta de mecanismos de cabildeos que permitan mantener una voz de enfermería a nivel gubernamental y de toma de decisiones.

\section{La construcción de una visión de enfermería}

Mario Rovere en su artículo en Educación Médica y Salud de abril/junio de 1992, sobre "Aportes para una Metodología de Planificación Estratégica de Recursos Humanos en Salud" al referirse al momento de la visión, afirma: "Se busca una definición responsable y consciente de una situación a alcanzar que sirva como indicador eficaz y motivador colectivo de los esfuerzos que se realicen". La representación que llamamos futuro, tiene una poderosa influencia sobre nuestro presente, ya que dirige el sentido de nuestras acciones ${ }^{8}$.

Dos artículos recientes aparecidos en la revista Nursing Outlook hablan sobre una visión de enfermería, diciendo:

"El futuro de un nuevo paradigma de enfermería envuelve un riesgo quizás por encima de lo seguro que es seguir hablando siempre entre enfermeras"'.

"Una orientación prospectiva no deduce el futuro del presente pero empieza a imaginarse varias utopías realistas que son compatibles con el presente"'.

Aunque es probablemente presuntuoso ofrecer predicciones sobre el futuro de enfermería, estaremos mejor preparados para movernos hacia el próximo siglo si estamos informados acerca del pasado, hemos analizado el presente y nos hemos formado una visión del futuro.

\section{La práctica}

Las enfermeras tienen muchas fortalezas y oportunidades que pueden ser capitalizadas dentro de un nuevo paradigma que se construye no desde los parámetros de los paradigmas tradicionales sino alrededor de puntos de contacto interprofesional y comunitario. Los conceptos de salud, de cuidado primario, sistemas locales de salud, y promoción de la salud nos dan el derrotero para un nuevo paradigma. Un nuevo 
paradigma que entiende a salud como parte importante del desarrollo dentro de un enfoque socio ambiental dentro del concepto de atención primaria que envuelve ciertos principios fundamentales como son distribución equitativa, participación comunitaria, tecnología apropiada, un enfoque de promoción e prevención y una direccionalidad multisectorial ${ }^{10}$.

En un documento de trabajo sobre "El papel de enfermería en cuidado de salud del adulto", su autora, Edilma Guevara, afirma: "...las enfermeras de atención primaria están en una posición única para identificar los riesgos potenciales y promover la salud entre las poblaciones que ellas atienden. Esto se debe a la posición que ellas ocupan dentro del sistema de salud y su preparación como educadoras. Sin embargo, las enfermeras necesitan integrar el conocimiento de la historia natural de las enfermedades y de los factores de riesgo para cada sexo de acuerdo al ciclo de la vida. Además, las enfermeras necesitan conocer las características epidemiológicas y sociales de la comunidad para planear el cuidado de la gente sana. Entonces las enfermeras deben aprender a aplicar el enfoque epidemiológico en la evaluación del cuidado primario y en el desarrollo de investigación en la comunidad. Además, las enfermeras necesitan desarrollar una capacidad analítica y evaluativa que permita reconocer los aspectos sociales involucrados en los problemas de salud de la comunidad y las reglas de comunicación existentes en la comunidad"11.

En la nueva propuesta la organización de servicios como son los SILOS, enfermería ocupa un lugar preponderante para mostrar su decidida contribución dentro de la estrategia de atención primaria, bien sea como colaboradora de acciones en la comunidad, como promotora de la salud de las poblaciones a las que presta atención, como oferente de un seguimiento y cuidado continuo a personas con problemas, como proveedora de un cuidado integral de alta calidad en fases agudas e intensas de la enfermedad. Su rol funcional le permite, además, la participación en la elaboración de las políticas de atención así como la elaboración de un plan de trabajo com la comunidad y con individuos en particular.

Yendo más allá de un rol institucional, enfermería, en el futuro, podrá desarrollar diversas formas de lo que se podría llamar trabajo liberal, ligándose el trabajo comunitario o a la provisión de servicios como son la salud ocupacional, cuidado en casa, centros de día ancianos, o seguimiento de personas con enfermedades crónicas, cuidados intermedios para pacientes dados de alta tempranamente, y servicios de promoción de salud y manutención de la salud o sencillamente convirtiéndose en "defensor" de la comunidad para la solución conjunta de problemas, en general, y de salud en particular, dentro de un marco interinstitucional. Las enfermeras en conjunto con otras profesionales deben conocer a fondo algunas estrategias que permitan orientar su acción hacia la promoción de la salud. La Carta de Ottawa definió "promoción de la salud" como el proceso de darle la oportunidad a la gente de aumentar el control sobre salud y ambiente y mejorar su propia salud. Las estrategias para lograr dicho cometido se orientan a promover la motivación a través de: estimular la motivación personal, formación de grupos, organización comunitaria, mecanismos de coalición y acción política.

Dentro del nuevo paradigma, el liderazgo en enfermería cambia de un modelo centralizado a uno descentralizado en donde las decisiones son compartidas dentro de una administración participativa que, en vez de ejercer poder, ofrece poder; propiciando una actuación responsable. La Dra. Anne Baker en su artículo "Emergiendo un nuevo paradigma de liderazgo", afirma: "Liderazgo transformador significa un cambio en donde propósitos del líder y del seguidor son los mismos, creando unidad de criterios y propósitos colectivo. En este tipo de liderazgo, el líder y el seguidor crecen y se desarrollan conjuntamente". Afirma, también, que existen cuatro estrategias para concretar en la realidad este concepto de liderazgo. Estas son definición de una visión, creación de una arquitectura social, confianza organizacional y autoestima. Refiriéndose a la visión, se relaciona con la meta de la organización que debe ser clara, realista, optimista y compartida por todos. La arquitectura social se refiere a los modos de relación en la institución que deben ser construidos de tal forma que al mismo tiempo que permitan trabajo en equipo también provean la oportunidad para el desarrollo individual. La necesidad de una confianza institucional tanto del líder como del seguidor son indispensables en este 
tipo de liderazgo para que las relaciones individuales y grupales florezcan y conduzcan a la creatividad, innovación, poder para la toma de decisiones, seguridad y autoestima ${ }^{12}$.

\section{La investigación}

La investigación constituye un elemento fundamental en la Jacqueline Fawcett en su artículo "La Investigación y su Relevancia para la Práctica de Enfermería". El avance de la disciplina de enfermería e la práctica de enfermería recae en el desarrollo de un distinto conocimiento acerca de fenómenos relevantes. Sin embargo para ser de utilidad a la práctica de enfermería su conocimiento tiene que ser validado en la realidad concreta y solamente a través de la investigación se puede lograr esto. Enfatiza, además la importancia de la utilización de modelos de enfermería como guía para la investigación de enfermería, prosigue diciendo que la investigación actual adolece de una perspectiva de enfermería que podría darse si se usaran modelos conceptuales como marcos organizativos del estudio de investigación ${ }^{13}$.

\section{La educación}

Es necesario crear nuevos modelos de currículo en enfermería que permita a los graduados ser más responsables de las necesidades de la sociedad, más exitosos en humanizar la atención de salud dentro de un medio ambiente que cada día se tecnifica más. Un currículo orientado hacia un cuidado humanizado con mayor fortaleza acerca de problemas éticos y morales, más creativo, más capaz de propiciar el pensamiento crítico de abordar científicamente los problemas del paciente o comunidad y de defender los derechos del usuario ${ }^{14}$. De acuerdo a Reich una de las habilidades más necesitadas en el futuro será la simbólica analítica. En lugar de un énfasis en la transmisión de información, el foco será en el juico y la interpretación. Se les enseñará a los estudiantes a analizar e interpretar los datos que leen. Se les preguntará por los hechos seleccionados y su importancia, como llegaron a deducirlos y el porqué pueden ser contradictorios. Los estudiantes aprenden a analizar realidades desde diferentes ángulos $\mathrm{y}$, en consecuencia, a visualizar nuevas posibilidades y escogencias. Gustavo de Roux y colaboradores en su artículo "Participación Social y Sistemas locales de Salud" manifiesta que el proceso de enseñanza aprendizaje debe lograrse recuperando el espacio comunitario como escenario de aprendizaje para enriquecerlo con concepciones que le dan valor a la educación como un proceso de participación social. Debe insistirse, por otra parte, en que la población es el recurso potencial más importante que tiene cualquier país para cuidar su salud y, por lo tanto, dársele información y conocimiento para no ocurra el saber en unos y la ignorancia en otros. Además debe llevarse a la población la comprensión de la necesidad en salud e la elección de medios destinados a satisfacerla ${ }^{15}$. Es importante entonces que la enfermera proponga nuevos modelos colaborativos en donde se demuestre la participación de la comunidad organizada dentro de los diferentes estamentos de la atención u su contribución en el desarrollo del modelo. Las instituciones formadoras por su lado deben propender a que el estudiante profundice más en el área de ciencias sociales y de comunicación social, planificación urbana, que le permitan un mejor conocimiento de la población y de las dinámicas de la organización y participación social.

Además, como lo manifiesta Collado en su artículo "New models of Health Care", las instituciones educacionales tienen un gran potencial en el mejoramiento de los servicios. Los servicios, por otro lado, pueden contribuir grandemente a que la educación sea más relevante, los esfuerzos de unos y otros se pueden poner en 
movimiento en beneficio de educación y servicios ${ }^{16}$. El explorar posibilidades de colaboración entre educación y servicio y en donde la investigación juegue un papel preponderante llevaría a verdaderas innovaciones en el diseño de los servicios de salud.

\section{Los gremios y ONGs}

Los gremios, asociaciones y organismos no-gubernamentales serán un elemento fundamental para el desarrollo de enfermería. Los primeros reconociendo el valor inherente de la diversidad y la importancia de un cambio en la orientación de la práctica individualista a la de una colectiva y colaborativa, trabajando en ofrecer poder a las enfermeras, creando ambientes favorables para el trabajo, promoviendo legislaciones equitativas, propiciando posicionamientos ampliamente consultadas por las mayorías sobre aspectos que atañen a la salud de la población, propiciando la discusión de los aspectos éticos como base de una práctica responsable y según los centros colaboradores se convierten en verdaderos puntos focales para el desarrollo del conocimiento y su validación en la práctica, propiciándose el desarrollo proyectos estratégicos de impacto, posibilitando la educación de profesores y personal de servicio con una visión nueva de su misión, un entendimiento diferente de lo que es salud y capaz de hacer nuevas alianzas. Asimismo son un medio para expandir la cooperación técnica con los países que permitan la consecución de las metas de Salud para Todos.

E resumen podríamos decir que estamos en el momento adecuado para reflexionar sobre nuestro futuro - futuro que permita a enfermería involucrarse en las decisiones que atañen la salud de nuestros pueblos, tener la posibilidad de controlar nuestra propia práctica y enfatizar más en áreas problema de mayor impacto.

\section{Proyectos y programas}

Uno de los grandes proyectos en que enfermería ha venido trabajando en los países es el análisis prospectiva. Esto ha permitido analizar la situación actual (desde el punto de vista del pasado, presente y futuro), construir una imagen-objetivo a alcanzar y dentro de este derrotero identificar algunos problemas y elaborar proyectos y programas. Creo que la mayoría de los países ha llegado a tener un buen análisis de su situación, han identificado una serie de problemas. Estos problemas identificados son el puente para iniciar un proceso de cambio que se comienza con la construcción de proyectos y programas factibles de operacionalizar. Tal vez un aspecto muy importante a tener en cuenta en enfermería es la construcción de la viabilidad de dichos proyectos. Es decir el momento estratégico en donde necesitamos encontrar, descubrir o diseñar movimientos capaces de acercarnos o poner los objetivos a nuestro alcance. El descubrimiento y diseño de estrategias lleva implícito el análisis de oponentes, obstáculos, fortalezas, debilidades y oportunidades. Es necesario entonces que dentro de la discusión en enfermería se plantee como podemos hacer que los proyectos necesarios se conviertan en posibles teniendo en cuenta lo anteriormente dicho.

A un nivel global me atrevería a sugerir cinco grandes proyectos:

1. La contribución de enfermería a la salud y desarrollo.

2. Construcción de un liderazgo en enfermería. Una nueva imagen en enfermería, (educación, práctica, gremios). Formación avanzada en salud pública, gerencia de servicios, áreas prioritarias de enfermería y salud pública. 
3. La formación de enfermería: nuevas dimensiones y propuestas educativas.

4. Desarrollo de la capacidad de investigación.

5. La contribución de organismos internacionales, ONG, centros colaboradores, sociedades científicas, asociaciones al desarrollo de la educación y la práctica de enfermería y de la salud de la población.

La planificación estratégica advierte sobre los análisis que dan por supuesto la existencia de altas concentraciones de poder que pueden transformar fácilmente lo planificado siendo que en la práctica la principal restricción es la de que el poder se encuentra fragmentado. Por eso es importante en enfermería dentro del análisis, identifique actores institucionales y fuerzas sociales que participan en la construcción del campo de acción de enfermería en un determinado país. Es dentro de este ambiente conflictivo e incierto, que los diversos actores sociales se mueven, y es en relación a sus lecturas de la realidad que se proponen las intervenciones que se suponen más ventajosas. El estudio de las fuerzas de poder, situaciones en conflicto, o de influencia es importante si queremos hacer planteamientos estratégicos viables. Es decir, la planificación estratégica interna identificar no a partir del debe ser sino a partir de como es y su relación con lo deseado, las estrategias más adecuadas para la intervención exitosa de diferentes fuerzas sociales $^{17}$.

La planificación estratégica advierte que en los espacios sociales el impulso transformador no surge de arriba a abajo o de abajo hacia arriba sino desde donde se puede, desde donde de generan las propuestas más innovadoras o las estrategias más exitosas ${ }^{18}$. Creo que esto es fácilmente observable en enfermaría donde surgen propuestas innovadoras o transformadoras desde instituciones o grupos por fuera de las estructuras burocráticas establecidas.

Creo haber presentado a ustedes un panorama general de enfermería dentro de la región conciliando algunos aspectos del pensamiento estratégico. Espero que a través de lecturas e intercambio con expertos Uds. puedan aclarar muchos puntos y seguir profundizando sobre el tema pero, quizás, lo más importante es que Uds. construyan su propio análisis identificando los problemas, determinando proyectos y construyendo una visión de enfermería que las lleve a determinar planes y proyectos que sean viables y factibles de llevarse a cabo.

Es por lo tanto necesario que enfermería parta de una visión totalizadora de la realidad en la búsqueda y fortalecimiento de su saber y su práctica, que integre una postura crítica en sus acciones y que busque en su hacer unas praxis transformadora que coadyude a conseguir una sociedad latinoamericana más justa, más libre y más sana.

Nursing in Latin America is facing a number of serious challenges significantly effecting contributions its personnel con make to the health of the people they serve.

This article presents an overview of nursing as an important component of the health system in Latin America through the prospective and orientation provided by strategic planning.

The author, following an analysis of the nursing in the Region, identifies several problems and then creates a vision for the future. The article looks at the possibilities of developing and implementing projects and action plans to address the priority problems. It sees strategic thinking as a possible means for nursing to reflect on and maximize its contribution and presence in the solution of the health problems in the current decade and into the next century.

\section{REFERÊNCIAS BIBLIOGRÁFICAS}

01. SOUZA, Alina. Tesis de grado. Desarrollo de los Servicios de Enfermería en la OPS: Impacto en la Educación de Enfermería en la América Latina 1940-1980. Ohio State University. 1982. 
02. ORGANIZACIÓN PANAMERICANA DE LA SALUD. Cuarenta años de cooperación técnica de la OPS en América Latina. Washington, 1983. Documento presentado en la reunión regional del grupo consultor sobre educación en enfermería.

03. ORGANIZACIÓN PANAMERICANA DE LA SALUD. Orientaciones estratégicas y prioridades programáticas de la Organización Panamericana de la Salud en el cuadrienio 1991-1994. Washington, 1990. Documento presentado na 23. Conferencia Sanitaria Panamericana 42. Reunión del Comité Regional.

04. ORGANIZACIÓN PANAMERICANA DE LA SALUD. La cooperación técnica en el contexto de la crisis de desarrollo de recursos humanos. 1990.

05. ORGANIZACIÓN PANAMERICANA DE LA SALUD. Estudio sobre programas de formación de enfermeras profesionales en América Latina - 1984. (Serie de Desarrollo de Recursos Humanos, n. 61).

06. MALHER, Halfdan. Discursos de inauguración conferencia "Liderazgo en Enfermería hacia salud para todos: un reto y una estrategia para la acción". Tokyo, 1986.

07. MANGAY-MANGLACAS, Almeida. Nursing in developing countries: needs and prospects. J.Adv.Nurs, Oxford, v. 17 p. 267-270, 1992

08. ROVERE, Mario. Capacitación avanzada en el desarrollo de recursos humanos: aportes para una metodología de planificación estratégica de recursos humanos en salud. Educ.Méd.Salud, Washington, v. 2, abr./jun. 1992.

09. SHULTZ, Jane et al. Growing edges of a new paradigm: the future of nursing in the health of the nation.

10. REGISTER NURSES ASSOCIATION OF BRITISH COLUMBIA. Determinants of health: empowering strategies for nursing practice: a background paper. 1992.

11. GUEVARA, Edilma. El papel de enfermería en el cuidado del adulto en énfasis en la promoción de la salud. Washington, 1991.

12. BAKER, Anne M. An emerging leadership paradigm: nursing and health care.

13. FAUCETT, Jackeline. La investigación y su relevancia para la práctica de enfermería McGraw Hill, 1983. Cap. 14: the nursing profesion: a time to speak.

14. BEVIS, Olivia, WESTON, Jean. Toward a caring curriculum: a new pedagogy for nursing. New York, NLN, 1989. P.28-48 (National League of Nursing, 15-2278).

15. DE ROAUX, Gustavo. Participación social y sistemas locales de salud. Washington, OPS/OMS, 1990. P. 28-48 (Publicación Científica, n. 519).

16. COLLADO, Carol. New models in health care. In Primary Health Care and Local Health Systems in Caribbean, PAHO, 1989.

17. ROVERE, Mario. IBID

18. ROVERE, Mario. IBID 